\title{
FÓSFORO EXTRAÍDO POR MEHLICH I E RESINA DE TROCA ANIÔNICA EM SOLOS SUBMETIDOS Á CALAGEM
}

\author{
PHOSPHORUS EXTRACTED BY MEHLICH I AND ANION EXCHANGE RESIN IN \\ SOILS SUBJECTED TO LIMING
}

\section{Everton Martins ARRUDA ${ }^{1}$; Regina Maria Quintão LANA ${ }^{2}$; Hamilton Seron PEREIRA ${ }^{2}$}

1. Doutorando em Agronomia, Universidade Federal de Goiás - UFG, Goiânia, GO, Brasil, arruda.solos@ gmail.com; 2. Professor(a), Doutor(a),Instituto de Ciências Agrárias, Universidade Federal de Uberlândia - UFU, Uberlândia, MG, Brasil.

\begin{abstract}
RESUMO: Utilizou-se um Latossolo Vermelho distroférrico (LVdf) e um Neossolo Quartzarênico Órtico (RQo) que receberam diferentes doses de fósforo (P) e calcário, com o objetivo de avaliar o teor de fósforo disponível nos extratores Mehlich I e Resina de Troca Aniônica em condições de laboratório. O delineamento experimental foi em blocos casualizados em esquema fatorial consistindo de dois solos, três doses de fósforo na forma de superfosfato triplo $(0,150$ e $\left.300 \mathrm{~kg} \mathrm{ha}^{-1}\right)$ e três condições de correção do solo $\left(1 . \mathrm{CaCO}_{3}\right.$ aos 30 dias antes da aplicação do fósforo; $2 . \mathrm{CaCO}_{3}$ junto com a aplicação do fósforo e; 3 . Sem $\mathrm{CaCO}_{3}$ ) calculadas para elevar a saturação por bases a $70 \%$, com 4 repetições, totalizando 72 parcelas. $\mathrm{O}$ experimento teve duração de 150 dias, marcando o fim do ciclo de reações químicas, sendo realizadas amostragens aos 30, 60, 90 e 120 dias após a aplicação do fósforo. Foram avaliados o pH, P-Mehlich, P-Resina e Premanescente. Em ambos os solos, os valores de $\mathrm{pH}$ aumentaram perante o uso do $\mathrm{CaCO}_{3}$. No LVdf os teores de $\mathrm{P}$ extraídos pelo método Mehlich I foram inferiores nos tratamentos que estiveram sob prática da calagem, sendo contrários aos obtidos pelo método da Resina de Troca Aniônica, que se mostraram superiores com a aplicação do $\mathrm{CaCO}_{3}$. O RQo apresentou resultados semelhantes ao LVdf, mostrando que a extração com Resina de Troca Aniônica detecta com maior eficiência os aumentos nos teores de fósforo disponível perante aplicação de $\mathrm{CaCO}_{3}$.
\end{abstract}

PALAVRAS-CHAVE: Adsorção. Calcário. Incubação. Extratores;

\section{INTRODUÇÃO}

Em razão de maximizar a utilização de fósforo $(\mathrm{P})$ na agricultura, o empresário rural tem procurado na amostragem e na interpretação dos resultados das análises químicas dos solos seus principais vínculos de informações no manejo da adubação fosfatada.

A análise química do solo demonstra a quantificação do fósforo disponível na solução do solo, sendo realizada por métodos analíticos através de soluções extratoras, podendo ser via dissolução ácida, troca iônica, complexação e/ou hidrolise de cátions (GATIBONI et al., 2003). O grande número de métodos em uso para avaliação deste elemento, de certa forma, já é um indicativo da complexidade do seu comportamento no solo (LARSEN, 1967). Apesar dos vários métodos de extração do nutriente no solo, nem sempre os valores obtidos nas análises químicas se correlacionam com as quantidades absorvidas pelas plantas. Dentre os métodos mais utilizados no Brasil, se destacam Mehlich I (MEHLICH, 1978) e Resina de Troca Aniônica (RAIJ et al., 1986).

O método Mehlich I tem sido o mais empregado ultimamente em nosso país, dada a sua viabilidade prática e econômica nas análises de rotina, à exceção do estado de São Paulo (ROSSI; FAGUNDES, 1998). Contudo, essa vantagem é apenas para o trabalho laboratorial e para determinação considerada de forma isolada, pois a eficiência de extração do $\mathrm{P}$ por este método sofre grande influência da capacidade tampão de fosfatos do solo (SANTOS, 2009), que é uma medida da capacidade do solo em manter um nível determinado de P na solução (BECKETT; WHITE, 1964; SILVA; RAIJ, 1999). Entretanto, o método da Resina de Troca Aniônica já tem apresentando resultados satisfatórios em relação a outros extratores. Este método fundamenta-se na premissa de simular o comportamento do sistema radicular das plantas na absorção de fósforo do solo (RAIJ, 1978). Outro fator que auxilia na quantificação do elemento no solo é o fósforo remanescente, que é uma medida estreitamente correlacionada à capacidade máxima de adsorção e à capacidade tampão de fosfatos (ALVAREZ; FONSECA, 1990; ALMEIDA et al., 2003).

Apesar do $\mathrm{P}$ disponível em diferentes extratores ser considerado uma medida de quantidade, o fósforo extraído refere-se apenas á uma fração desse valor, podendo esta fração variar de um solo para outro (NOVAIS et al., 2007). No entanto, uma característica importante na escolha de 
métodos de determinação de $\mathrm{P}$ em solos é a sua aplicabilidade em diferentes tipos de solos, principalmente no que se refere ao $\mathrm{pH}$ (SILVA; RAIJ, 1999). O aumento do $\mathrm{pH}$ faz com que as cargas superficiais das partículas do solo tornam-se cada vez mais negativas, aumentando a repulsão entre o fosfato e a superfície adsorvente, diminuindo assim o potencial eletrostático do plano de adsorção (HAYNES, 1984; BARROW, 1985).

$\mathrm{O}$ aumento dos valores de $\mathrm{pH}$ advindos do uso de corretivos na agricultura é uma prática que contribui para aumentar a disponibilidade de $\mathrm{P}$ e a eficiência dos fertilizantes fosfatados (SOUSA et al., 2004). No entanto, para conseguir boa disponibilidade deste elemento na solução do solo é importante o uso correto de combinações distintas de fosfatos e/ou corretivos de acidez.

O objetivo deste trabalho foi avaliar o fósforo disponível através dos extratores Mehlich I e
Resina de Troca Aniônica em solos do cerrado sob diferentes condições de calagem e doses de fósforo.

\section{MATERIAL E MÉTODOS}

O experimento foi realizado no Laboratório de Fertilidade do Solo e Nutrição de Plantas da Universidade Federal de Goiás, no ano de 2010. Foram utilizadas amostras previamente selecionadas de dois solos da região do sudoeste goiano, provenientes do município de Jataí-GO, na camada de $0-0,2 \mathrm{~m}$, sendo um Latossolo Vermelho distroférrico típico (LVdf) e um Neossolo Quartzarênico Órtico típico (RQo), conforme EMBRAPA (2013). As amostras de solo coletadas foram secas em estufa $\left(60^{\circ} \mathrm{C}\right)$, destorroadas e peneiradas. Na Tabela 1 são apresentadas as características físicas e químicas dos solos estudados.

Tabela 1. Caracterização física e química dos solos estudados.

\begin{tabular}{ccc}
\hline \multirow{2}{*}{ Propriedades físicas e químicas } & \multicolumn{2}{c}{ Solos $^{11}$} \\
\cline { 2 - 3 } & RQo & LVdf \\
\hline Argila $\left(\mathrm{g} \mathrm{kg}^{-1}\right)$ & 94,5 & 398,1 \\
Silte $\left(\mathrm{g} \mathrm{kg}^{-1}\right)$ & 22,6 & 140,2 \\
Areia fina $\left(\mathrm{g} \mathrm{kg}^{-1}\right)$ & 534,9 & 337,0 \\
Areia grossa $\left(\mathrm{g} \mathrm{kg}^{-1}\right)$ & 348,0 & 124,7 \\
Matéria orgânica $\left(\mathrm{g} \mathrm{kg}^{-1}\right)$ & 7,0 & 14,0 \\
$\mathrm{pH}\left(\mathrm{CaCl}_{2}\right)$ & 4,0 & 4,7 \\
$\mathrm{Ca}\left(\mathrm{cmol}_{\mathrm{c}} \mathrm{dm}^{-3}\right)$ & 0,1 & 0,1 \\
$\mathrm{Mg}\left(\mathrm{cmol}_{\mathrm{C}} \mathrm{dm}^{-3}\right)$ & 0,0 & 0,1 \\
$\mathrm{H}+\mathrm{Al}\left(\mathrm{cmol}_{\mathrm{C}} \mathrm{dm}^{-3}\right)$ & 1,9 & 2,8 \\
$\mathrm{AI}\left(\mathrm{cmol}_{\mathrm{C}} \mathrm{dm}^{-3}\right)$ & 0,6 & 0,1 \\
$\mathrm{~K}\left(\mathrm{mg} \mathrm{dm}^{-3}\right)$ & 24 & 19 \\
P (mehlich) $\left(\mathrm{mg} \mathrm{dm}^{-3}\right)$ & 1,0 & 0,5 \\
CTC $\left(\mathrm{cmolo} \mathrm{dm}^{-3}\right)$ & 2,2 & 3,05 \\
Saturação de Bases $(\%)$ & 12,0 & 8,2 \\
\hline RQo = Neossolo Quartzarênico Órtico típico. LVdf = Latossolo Vermelho Distroférrico típico (EMBRAPA, 2013).
\end{tabular}

Os tratamentos foram dispostos em delineamento em blocos casualizados (DBC) propondo um esquema fatorial consistindo de dois solos, três doses de fósforo na forma de superfosfato triplo com $43 \%$ de $\mathrm{P}_{2} \mathrm{O}_{5},\left(0,150\right.$ e $\left.300 \mathrm{~kg} \mathrm{ha}^{-1}\right)$ e três condições de correção do solo, sendo, o carbonato de cálcio puro $\left(\mathrm{CaCO}_{3}\right)$ aplicado 30 dias antes da aplicação do fósforo $(\mathrm{C} 30) ;$ o $\mathrm{CaCO}_{3}$ junto com a aplicação do fósforo ( $\mathrm{C} 0)$ e; sem aplicação de $\mathrm{CaCO}_{3}$ (SC), em doses calculadas para elevar a saturação por bases a $70 \%$, com 4 repetições, totalizando 72 parcelas. A dose de $\mathrm{CaCO}_{3}$ usada em cada solo foi $687 \mathrm{mg} \mathrm{kg}^{-1}$ e $950 \mathrm{mg} \mathrm{kg}^{-1}$ para o RQo e LVdf, respectivamente.

Os materiais foram homogeneizados em potes plásticos com 150 gramas de solo a uma temperatura média de $26 \mathrm{C}^{\mathrm{o}}$, fechados e mantidos próximos à capacidade de campo com água destilada durante 120 dias, assim os solos RQo e LVdf receberam respectivamentes $41 \mathrm{~mL}$ e $57 \mathrm{~mL}$ de água destilada em cada pote com $150 \mathrm{~g}$ de solo. $\mathrm{O}$ tratamento que recebeu $\mathrm{CaCO}_{3}$ aos 30 dias antes da aplicação de fósforo foi incubado por 150 dias no total. Após aplicação do fósforo, em cada pote foi retirada cerca de 30 gramas de solo a cada 30 dias. As amostras de solo foram levadas à estufa durante 48 horas visando encerrar as reações químicas. Desta forma, foram realizadas quatro amostragens, aos 30, 60, 90 e 120 dias após a aplicação do fósforo (DAAP). Foram realizadas análises de $\mathrm{pH}$ em água na proporção de 1:2,5 ; fósforo remanescente (Prem) (ALVAREZ et al., 2000) para avaliar o teor de fósforo presente na solução do solo, além do fósforo disponível extraído pelo método Mehlich I (MEHLICH, 1978) e pelo método da Resina de Troca Aniônica (RAIJ et al., 1986). As análises 
estatísticas dos dados envolveram a análise de variância (Teste de F) e comparação de médias pelo teste de Tukey $(\mathrm{P}<0,05)$, através do programa SISVAR (FERREIRA, 2008).

\section{RESULTADOS E DISCUSSÃO}

$\mathrm{Na}$ amostragem realizada 30 dias antes da aplicação do fósforo (30 DAAP) no Latossolo Vermelho Distroférrico (LVdf) não foi verificado interação significativa, ocorrendo aumentos nos valores de $\mathrm{pH}$ apenas em função da própria condição de calagem estabelecida, não sendo, observado aumentos significativos para as doses de $\mathrm{P}$ utilizadas (Tabela 2).

$\mathrm{O} \mathrm{pH}$ da solução do solo foi influenciado pela aplicação de $\mathrm{CaCO}_{3}$ e dose de $\mathrm{P}$ a partir da segunda amostragem (60 DAAP), apresentando superioridade nos tratamentos com uso do corretivo junto a aplicação de $\mathrm{P}(\mathrm{C} 0)$ e 30 dias antes da aplicação de $\mathrm{P}$ (C30), decrescendo em relação as doses de fosfato.

$\mathrm{Na}$ última amostragem realizada $(120$ DAAP) maiores valores de $\mathrm{pH}$ foram verificados quando a calagem foi realizada 30 dias antes da aplicação de $\mathrm{P}$, notoriamente, este comportamento deve ter ocorrido devido ao modo de aplicação do $\mathrm{CaCO}_{3}$ anterior ao fosfato que teve um maior tempo para disponibilizar o cálcio para solução do solo. Todavia, a aplicação do $\mathrm{CaCO}_{3}$ juntamente com o fosfato diminui os valores de $\mathrm{pH}$, isto em função do cálcio do $\mathrm{CaCO}_{3}$ reagir com o $\mathrm{P}$ do fosfato e não com o próprio solo, ocasionando assim um problema de caráter analítico.

Tabela 2. Valores de $\mathrm{pH}\left(\mathrm{H}_{2} \mathrm{O}\right)$ em diferentes condições de calagem e doses de $\mathrm{P}_{2} \mathrm{O}_{5}$, em Latossolo Vermelho Distroférrico no município de Jataí-GO, 2010.

\begin{tabular}{|c|c|c|c|c|}
\hline $\begin{array}{c}\text { Tratamento } \mathrm{P}_{2} \mathrm{O}_{5} \\
\left(\mathrm{~kg} \mathrm{ha}^{-1}\right)\end{array}$ & 0 & 150 & 300 & Média \\
\hline \multicolumn{5}{|c|}{$\overline{\mathrm{pH}} \overline{\mathrm{panAM \textrm {D }}}$} \\
\hline $\mathrm{SC}$ & 5,42 & 5,39 & 5,58 & $5,46 \mathrm{c}$ \\
\hline $\mathrm{C} 0$ & 7,53 & 7,56 & 7,54 & $7,54 \mathrm{a}$ \\
\hline C30 & 7,47 & 7,27 & 7,23 & $7,32 \mathrm{~b}$ \\
\hline \multirow{2}{*}{\multicolumn{5}{|c|}{$\begin{array}{c}\text { 6,74 A } \\
\text { Amostragem } 60 \text { DAAP }\end{array}$}} \\
\hline & & & & \\
\hline $\mathrm{C} 0$ & $\begin{array}{l}, 15 \mathrm{DaO} \\
6,78 \mathrm{aA}\end{array}$ & $\begin{array}{l}5,06 \mathrm{cB} \\
6,60 \mathrm{bB}\end{array}$ & $\begin{array}{l}5,100 \mathrm{~A} \\
6,65 \text { a B }\end{array}$ & $\begin{array}{l}5,12 \mathrm{c} \\
6,68 \mathrm{~b}\end{array}$ \\
\hline $\mathrm{C} 30$ & $6,74 \mathrm{aB}$ & $6,83 \mathrm{aA}$ & 6,69 a B & $6,75 \mathrm{a}$ \\
\hline Média & $6,22 \mathrm{~A}$ & $6,16 \mathrm{~B}$ & $6,17 \mathrm{AB}$ & \\
\hline \multicolumn{5}{|c|}{ Amostragem 90 DAAP } \\
\hline $\mathrm{SC}$ & $5,12 \mathrm{cA}$ & $4,91 \mathrm{bAB}$ & $4,82 \mathrm{~b} \mathrm{~B}$ & $4,95 \mathrm{c}$ \\
\hline $\mathrm{C} 0$ & $6,61 \mathrm{bA}$ & $6,57 \mathrm{aA}$ & 6,52 a A & $6,57 \mathrm{~b}$ \\
\hline $\mathrm{C} 30$ & $7,26 \mathrm{aA}$ & $6,84 \mathrm{aB}$ & 6,49 a C & $6,86 \mathrm{a}$ \\
\hline Média & $6,33 \mathrm{~A}$ & $6,11 \mathrm{~B}$ & $5,94 \mathrm{C}$ & \\
\hline \multicolumn{5}{|c|}{ Amostragem 120 DAAP } \\
\hline $\mathrm{SC}$ & $4,81 \mathrm{cA}$ & $4,61 \mathrm{cB}$ & 4,40 с C & $4,61 \mathrm{c}$ \\
\hline $\mathrm{C} 0$ & $6,86 \mathrm{bA}$ & $6,85 \mathrm{bA}$ & $6,79 \mathrm{~b} \mathrm{~A}$ & $6,83 \mathrm{~b}$ \\
\hline $\mathrm{C} 30$ & $7,26 \mathrm{aA}$ & $7,10 \mathrm{aB}$ & 6,95 a C & $7,10 \mathrm{a}$ \\
\hline Média & $6,31 \mathrm{~A}$ & $6,19 \mathrm{~B}$ & $6,04 \mathrm{C}$ & \\
\hline
\end{tabular}

${ }^{\pi}$ Calagem, onde, $\mathrm{SC}=$ Sem calagem. $\mathrm{C} 0=$ Calagem realizada junto a aplicação do fósforo. $\mathrm{C} 30=$ Calagem realizada 30 dias antes da aplicação do fósforo. DAAP= Dias após aplicação do fósforo. * Médias seguida de letras distintas minúsculas na coluna e maiúsculas na linha diferem entre si pelo teste de Tukey $(\mathrm{P}<0,05)$.

No LVdf os valores de P disponível obtidos pelo extrator Mehlich I foram inferiores nos tratamentos que estiveram sob condições de calagem ( $\mathrm{C} 0$ e $\mathrm{C} 30)$, evidenciando assim, certa ineficiência deste método em quantificar o $\mathrm{P}$ da solução do solo sobre efeito da prática da calagem. Os menores níveis de $\mathrm{P}$ disponível foram observados nas amostragens realizadas 60 DAAP e aos 90 DAAP, nas doses de 150 e/ou $300 \mathrm{~kg} \mathrm{ha}^{-1}$ de $\mathrm{P}_{2} \mathrm{O}_{5}$, sendo ainda que, onde os tratamentos não predicavam aplicação de $\mathrm{CaCO}_{3}$ (SC), os valores para $\mathrm{P}$ disponível foram significamente superiores (Tabela 3).

A máxima disponibilidade de $\mathrm{P}$ na solução do solo encontra-se com pH em torno de 6,0 (OLSEN; KHASAWNEH, 1980). Entretanto, o aumento do $\mathrm{pH}$ da solução do solo no LVdf para valores próximos á 6,0 não foi capaz de revelar maior disponibilidade de fósforo. 
Tabela 3. Teores de Fósforo (Mehlich I) em diferentes condições de calagem e doses de $\mathrm{P}_{2} \mathrm{O}_{5}$, em Latossolo Vermelho Distroférrico no município de Jataí-GO, 2010.

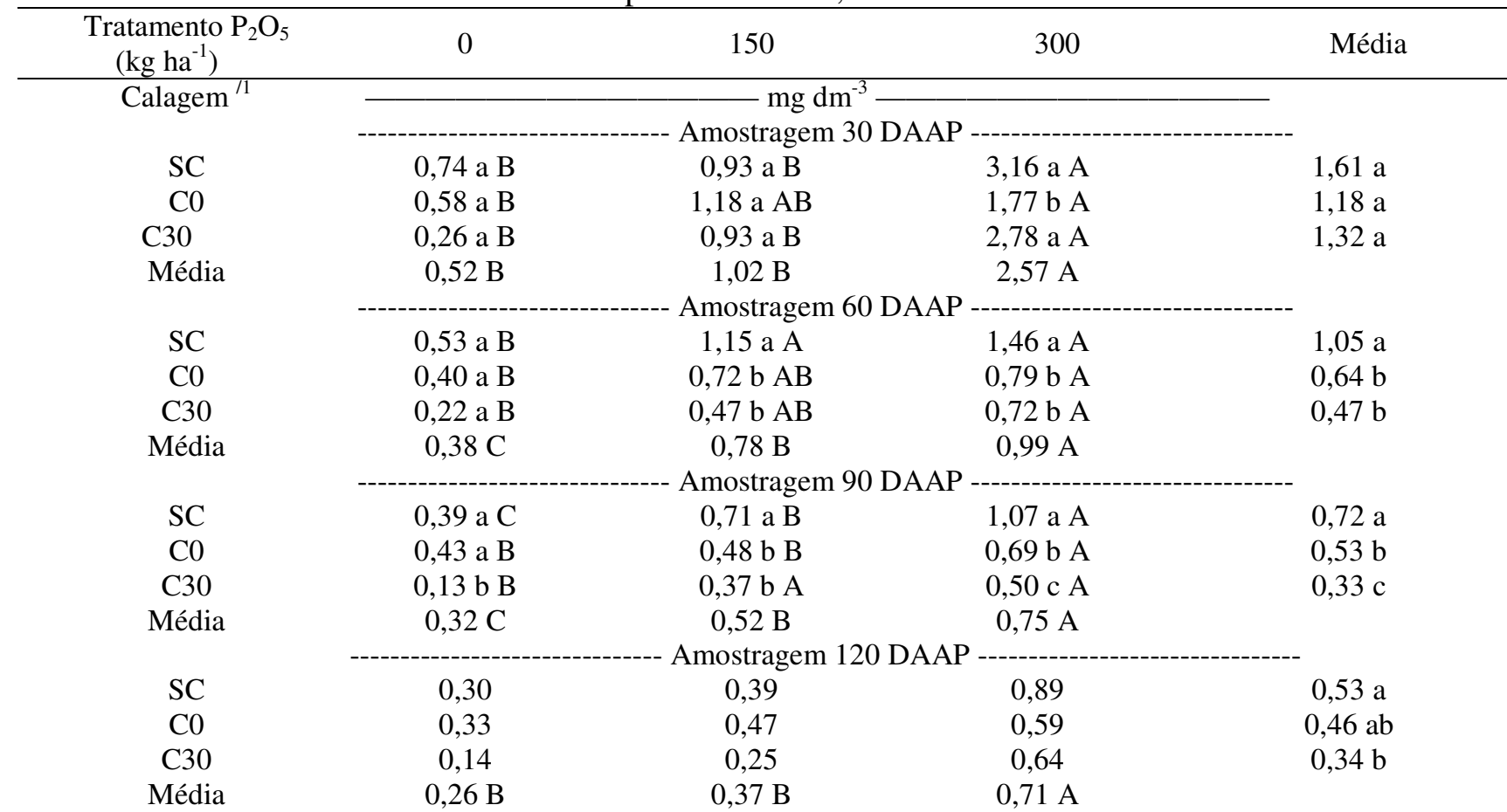

${ }^{1}$ Calagem, onde, $\mathrm{SC}=$ Sem calagem. $\mathrm{C} 0=$ Calagem realizada junto a aplicação do fósforo. $\mathrm{C} 30$ = Calagem realizada 30 dias antes da aplicação do fósforo. DAAP= Dias após aplicação do fósforo. * Médias seguida de letras minúsculas na coluna e maiúsculas na linha, não diferem entre si pelo teste de Tukey $(\mathrm{P}<0,05)$.

De acordo com Novais et al., (2007) para Mehlich I, valores subestimados do P disponível tem sido verificados, com frequência, em solos argilosos, de modo especial naqueles com $\mathrm{pH}$ mais elevado, em razão de ter seu poder de extração desgastado pelo próprio solo.

Raij e Quaggio (1990) encontraram resultados semelhantes quando relacionaram $\mathrm{pH}$ e $\mathrm{P}$ disponível com quatro experimentos de campo, onde o $\mathrm{P}$ determinado pelo extrator Mehlich I não indicou acréscimos pelo efeito do $\mathrm{CaCO}_{3}$. A interferência da calagem nos teores de $\mathrm{P}$ disponível é atribuída ao processo de fixação do fósforo ao cálcio proveniente do $\mathrm{CaCO}_{3}$ que ainda não reagiu com a solução do solo, além do que é liberado pelo próprio extrator ácido.

A calagem oferta grande quantidade de cálcio a solução do solo, fazendo com que parte do fósforo disponível seja "fixado", diante disso, a eficiência do extrator é afetada. De acordo com Novais e Smyth (1999) este maior teor de cálcio trocável pode promover a precipitação do $\mathrm{P}$, formando fosfatos bi e tricálcicos de baixa solubilidade, reduzindo os valores de $\mathrm{P}$ extraídos pelo método Mehlich I.

Os valores das médias de $\mathrm{P}$ extraídos pelo método da Resina de Troca Aniônica aumentaram com os valores de pH (Tabela 4), apresentando assim, comportamento contrário aos obtidos pelo extrador Mehlich I.

Nas amostragens realizadas aos 60 DAAP, 90 DAAP e 120 DAAP foram observados aumentos nos valores das médias de $\mathrm{P}$ na solução do solo nos tratamentos mantidos em condições de calagem $(\mathrm{C} 0$ e C30), expondo adequadamente o efeito da calagem em aumentar a disponibilidade de $\mathrm{P}$ neste solo. Este método mostrou não sofrer influências pelo corretivo de acidez do solo, o que também foi demonstrado por Raij et al., (1984).

A extração de $\mathrm{P}$ pelo método da Resina de Troca Aniônica, através da saturação com íon bicarbonato, proporciona certa estabilidade ao $\mathrm{pH}$ do meio, como se fosse um tamponamento induzido, mantendo o ambiente da solução do solo com valores constantes, próximos a faixa de maior disponibilidade do nutriente. Entretanto, a extração por Mehlich I, que faz uso de soluções químicas de carácter ácido, com pH em torno de 2,0 favorece os desequilíbrios das formas mais disponíveis de $\mathrm{P}$ no solo, subestimando seus níveis na solução do solo, que por consequência não consegue detectar $o$ aumento da disponibilidade de $\mathrm{P}$ oriundo da adição do $\mathrm{CaCO}_{3}$.

Resultados semelhantes foram encontrados por Camargo et al., (2010), em trabalho conduzido em casa de vegetação, onde obtiveram aumentos nos 
teores de P extraídos em Resina de Troca Aniônica com doses de $\mathrm{CaCO}_{3}$ em Latossolo Vermelho distrófico típico mantidos por 90 dias em fase de incubação.

Tabela 4. Teores de Fósforo (Resina) em diferentes condições de calagem e doses de $\mathrm{P}_{2} \mathrm{O}_{5}$, em Latossolo Vermelho Distroférrico no município de Jataí-GO, 2010.

\begin{tabular}{|c|c|c|c|c|}
\hline $\begin{array}{l}\text { Tratamento } \mathrm{P}_{2} \mathrm{O}_{5} \\
\quad\left(\mathrm{~kg} \mathrm{ha}^{-1}\right)\end{array}$ & 0 & 150 & 300 & Média \\
\hline Calagem $^{\prime 1}$ & \multicolumn{3}{|c|}{$\operatorname{mg~dm}^{-3}$} & \multirow[b]{2}{*}{$9,84 \mathrm{a}$} \\
\hline $\mathrm{SC}$ & 1,53 & 6,66 & 21,32 & \\
\hline $\mathrm{C} 0$ & 2,78 & 20,37 & 36,92 & $20,02 \mathrm{a}$ \\
\hline C30 & 1,70 & 10,59 & 28,26 & $13,52 \mathrm{a}$ \\
\hline Média & $2,00 \mathrm{~B}$ & $12,54 \mathrm{~B}$ & $28,83 \mathrm{~A}$ & \\
\hline & & mostrage & 891 & \\
\hline $\begin{array}{l}\mathrm{SC} \\
\mathrm{C} 0\end{array}$ & $\begin{array}{l}2,13 \\
2,95\end{array}$ & $\begin{array}{l}4,77 \\
8,90\end{array}$ & $\begin{array}{c}8,91 \\
11,66\end{array}$ & $\begin{array}{c}5,27 \mathrm{~b} \\
7,84 \mathrm{ab}\end{array}$ \\
\hline C30 & 4,59 & 5,93 & 14,89 & $8,47 \mathrm{a}$ \\
\hline Média & $3,23 \mathrm{C}$ & $6,53 \mathrm{~B}$ & $11,82 \mathrm{~A}$ & \\
\hline $\mathrm{SC}$ & 2,22 & $\begin{array}{l}\text { mostrage } \\
3,86\end{array}$ & 5,93 & $4,00 \mathrm{~b}$ \\
\hline $\mathrm{C} 0$ & 3,86 & 7,52 & 9,94 & $7,11 \mathrm{a}$ \\
\hline C30 & 2,53 & 6,06 & 11,84 & $6,81 \mathrm{a}$ \\
\hline Média & $2,87 \mathrm{C}$ & $5,82 \mathrm{~B}$ & $9,23 \mathrm{~A}$ & \\
\hline $\mathrm{SC}$ & 2,78 & $\begin{array}{l}\text { mostrager } \\
3,69\end{array}$ & 6,19 & $4,22 \mathrm{c}$ \\
\hline $\mathrm{C} 0$ & 7,70 & 13,17 & 15,50 & $12,12 \mathrm{a}$ \\
\hline $\mathrm{C} 30$ & 3,99 & 7,05 & 13,13 & $8,06 \mathrm{~b}$ \\
\hline Média & $4,82 \mathrm{C}$ & 7,97 B & $11,60 \mathrm{~A}$ & \\
\hline
\end{tabular}

${ }^{1}$ Calagem, onde, $\mathrm{SC}=$ Sem calagem. $\mathrm{C} 0=$ Calagem realizada junto a aplicação do fósforo. $\mathrm{C} 30=$ Calagem realizada 30 dias antes da aplicação do fósforo. DAAP= Dias após aplicação do fósforo. * Médias seguida de letras minúsculas na coluna e maiúsculas na linha, não diferem entre si pelo teste de Tukey $(\mathrm{P}<0,05)$.

Apenas na amostragem realizada aos 90 DAAP, os teores de P-remanescente (P-rem) apresentaram interação significativa entre as doses de $\mathrm{P}$ e as condições de calagem no LVdf, mostrando superioridade nos tratamentos onde não foi aplicado $\mathrm{CaCO}_{3}$ e as doses de $\mathrm{P}$ foram de 0 e/ou $150 \mathrm{~kg} \mathrm{ha}^{-1}$ (Tabela 5). Nas demais amostragens realizadas, os valores das médias revelaram níveis inferiores nos tratamentos onde houve aplicação de $\mathrm{CaCO}_{3}(\mathrm{C} 0 \mathrm{e}$ C30).

Estes resultados ocorreram devido ao uso do $\mathrm{CaCO}_{3}$ proporcionar maior capacidade de precipitação do $\mathrm{P}$ na solução do solo, expressado pela redução do valor do P-rem, principalmente onde foram obtidos altos valores de $\mathrm{pH}$, além é claro, da formação de formas bi e tricálcicas oriundas do cálcio do $\mathrm{CaCO}_{3}$.

Houve interação significativa entre as condições de calagem e as doses de $\mathrm{P}(\mathrm{P}<0,05)$ para os valores de $\mathrm{pH}$ observados no Neossolo quartzarênico Órtico (RQo). Os maiores valores de $\mathrm{pH}$ foram verificados nas aplicações de $\mathrm{CaCO}_{3}$, em C0 e C30 (Tabela 6).
Quando houve aumento nas doses de $\mathrm{P}$ utilizadas foram observados decréscimos nos valores de $\mathrm{pH}$ da solução do solo. Estes resultados verificados a partir da segunda amostragem (60 DAAP), evidencia que a força iônica adquirida na solução do solo diminuíram os valores de $\mathrm{pH}$.

Nos tratamentos onde a calagem foi realizada junto a aplicação do $\mathrm{P}(\mathrm{C} 0)$, foram obtidos maiores valores de $\mathrm{pH}$, este fato, foi verificado nas amostragens realizadas aos $60 \mathrm{DAAP}$, propriamente na dose de $300 \mathrm{~kg}^{-1}$ de $\mathrm{P}_{2} \mathrm{O}_{5}$ e também, nas amostragens aos 90 DAAP e 120 DAAP, tanto na dose de $150 \mathrm{~kg} \mathrm{ha}^{-1}$, quanto na dose de $300 \mathrm{~kg} \mathrm{ha}^{-1}$ de $\mathrm{P}_{2} \mathrm{O}_{5}$.

No RQo não foi observado diferença significativa na interação entre os valores obtidos de P extraídos por Mehlich I e as condições de calagem (Tabela 7). Através desta ineficiência entre os tratamentos, ficou evidenciado que o extrator não conseguiu quantificar os incrementos nos teores de $\mathrm{P}$ do solo quando foi feita aplicação de $\mathrm{CaCO}_{3}$. 
Tabela 5. Teores de Fósforo Remanescente em diferentes condições de calagem e doses de $\mathrm{P}_{2} \mathrm{O}_{5}$, em Latossolo Vermelho Distroférrico no município de Jataí-GO, 2010.

\begin{tabular}{|c|c|c|c|c|}
\hline $\begin{array}{l}\text { Tratamento } \mathrm{P}_{2} \mathrm{O}_{5} \\
\quad\left(\mathrm{~kg} \mathrm{ha}^{-1}\right)\end{array}$ & 0 & 150 & 300 & Média \\
\hline Calagem $^{/ 1}$ & \multicolumn{3}{|c|}{$-\mathrm{mg} \mathrm{dm}^{-3}$} & \\
\hline $\mathrm{SC}$ & 157 & $\begin{array}{l}\text { nostragen } \\
234\end{array}$ & 34 & $235 a$ \\
\hline $\mathrm{CO}$ & 1,11 & 1,15 & 1,42 & $1,22 \mathrm{~b}$ \\
\hline C30 & 0,36 & 0,48 & 1,13 & $0,66 \mathrm{c}$ \\
\hline Média & $1,01 \mathrm{~B}$ & $1,32 \mathrm{~B}$ & $1,90 \mathrm{~A}$ & \\
\hline $\mathrm{SC}$ & 1,18 & $\begin{array}{c}\text { Amostragen } \\
2.31\end{array}$ & 2.79 & $209 \mathrm{a}$ \\
\hline $\mathrm{CO}$ & 0,85 & 1,47 & 0,96 & $1,09 \mathrm{ab}$ \\
\hline $\mathrm{C} 30$ & 0,48 & 0,65 & 0,98 & $0,70 \mathrm{~b}$ \\
\hline \multirow[t]{2}{*}{ Média } & $0,83 \mathrm{~A}$ & $1,47 \mathrm{~A}$ & $1,56 \mathrm{~A}$ & \\
\hline & & Amostragen & -------- & \\
\hline $\mathrm{SC}$ & 1,62 a A & 1,19 a $\mathrm{AB}$ & 0,67 a B & $1,16 \mathrm{a}$ \\
\hline $\mathrm{C} 0$ & $0,43 \mathrm{~b} \mathrm{~A}$ & $0,75 \mathrm{ab} A$ & 0,82 a $A$ & $0,68 \mathrm{~b}$ \\
\hline C30 & $0,50 \mathrm{~b} A B$ & $0,31 \mathrm{~b} \mathrm{~B}$ & 1,20 a $\mathrm{A}$ & $0,67 \mathrm{~b}$ \\
\hline \multirow[t]{2}{*}{ Média } & $0,85 \mathrm{~A}$ & $0,75 \mathrm{~A}$ & $0,91 \mathrm{~A}$ & \\
\hline & & Amostragen & ------------ & \\
\hline $\mathrm{SC}$ & 1,57 & 3,17 & 0,73 & $1,82 \mathrm{a}$ \\
\hline $\mathrm{CO}$ & 0,55 & 0,44 & 0,75 & $0,58 \mathrm{~b}$ \\
\hline $\mathrm{C} 30$ & 0,63 & 1,34 & 1,38 & $1,12 \mathrm{ab}$ \\
\hline Média & $0,92 \mathrm{~A}$ & $1,65 \mathrm{~A}$ & $0,95 \mathrm{~A}$ & \\
\hline
\end{tabular}

Tabela 6. Valores de $\mathrm{pH}\left(\mathrm{H}_{2} \mathrm{O}\right)$ em diferentes condições de calagem e doses de $\mathrm{P}_{2} \mathrm{O}_{5}$, em Neossolo Quartzarênico Órtico no munícipio de Jataí-GO, 2010.

\begin{tabular}{|c|c|c|c|c|}
\hline $\begin{array}{r}\text { Tratamento } \\
\mathrm{P}_{2} \mathrm{O}_{5}\left(\mathrm{~kg} \mathrm{ha}^{-1}\right) \\
\end{array}$ & 0 & 150 & 300 & Média \\
\hline \multicolumn{5}{|l|}{ Calagem $^{/ 1}$} \\
\hline $\mathrm{SC}$ & $558 \mathrm{cB}$ & $568 \mathrm{~b} A \mathrm{~B}$ & $574 \mathrm{bA}$ & $566 c$ \\
\hline $\mathrm{CO}$ & $7,77 \mathrm{~b} \mathrm{~A}$ & 7,69 a A & 7,67 a $A$ & $7,11 \mathrm{~b}$ \\
\hline C30 & 8,02 a A & 7,83 a $\mathrm{B}$ & 7,78 a B & $7,88 \mathrm{a}$ \\
\hline Média & $7,12 \mathrm{~A}$ & 7,07 A & $7,06 \mathrm{~A}$ & \\
\hline & & mostragem & ----------- & \\
\hline $\mathrm{SC}$ & $5,30 \mathrm{~b} A$ & $5,20 \mathrm{bA}$ & $5,24 \mathrm{c} \mathrm{A}$ & $5,25 \mathrm{c}$ \\
\hline $\mathrm{CO}$ & 7,59 a $A$ & 7,58 a $\mathrm{A}$ & 7,37 a B & $7,52 \mathrm{a}$ \\
\hline C30 & 7,63 a $A$ & 7,41 a B & 6,98 b C & $7,34 \mathrm{~b}$ \\
\hline Média & $6,84 \mathrm{~A}$ & $6,73 \mathrm{~B}$ & $6,53 \mathrm{C}$ & \\
\hline & & Imostragem & & \\
\hline $\mathrm{SC}$ & $4,33 \mathrm{~b} \mathrm{~A}$ & $4,30 \mathrm{c} \mathrm{AB}$ & 4,12 c B & $4,25 \mathrm{c}$ \\
\hline $\mathrm{CO}$ & 7,69 a $A$ & 7,78 a $\mathrm{A}$ & 7,44 a B & $7,63 \mathrm{a}$ \\
\hline C30 & 7,64 a $A$ & 7,45 b B & $6,90 \mathrm{~b} \mathrm{C}$ & $7,33 \mathrm{~b}$ \\
\hline Média & $6,55 \mathrm{~A}$ & $6,51 \mathrm{~A}$ & $6,15 \mathrm{~B}$ & \\
\hline $\mathrm{SC}$ & $401 \mathrm{cA}$ & mostragem & $361 \mathrm{c} C$ & $379 \mathrm{c}$ \\
\hline $\mathrm{CO}$ & 7,89 a A & 7,96 a A & 7,71 a B & $7,85 \mathrm{a}$ \\
\hline $\mathrm{C} 30$ & $7,76 \mathrm{~b} \mathrm{~A}$ & $7,62 \mathrm{~b} \mathrm{~B}$ & $7,21 \mathrm{~b} \mathrm{C}$ & $7,53 \mathrm{~b}$ \\
\hline Média & $6,55 \mathrm{~A}$ & $6,45 \mathrm{~B}$ & $6,17 \mathrm{C}$ & \\
\hline
\end{tabular}

${ }^{1}$ Calagem, onde, $\mathrm{SC}=$ Sem calagem. $\mathrm{C} 0=$ Calagem realizada junto a aplicação do fósforo. $\mathrm{C} 30$ = Calagem realizada 30 dias antes da aplicação do fósforo. DAAP= Dias após aplicação do fósforo. * Médias seguida de letras minúsculas na coluna e maiúsculas na linha, não diferem entre si pelo teste de Tukey $(\mathrm{P}<0,05)$. 
Tabela 7. Teores de Fósforo (Mehlich I) em diferentes condições de calagem e doses de $\mathrm{P}_{2} \mathrm{O}_{5}$, em Neossolo Quartzarênico Órtico no munícipio de Jataí-GO, 2010.

\begin{tabular}{|c|c|c|c|c|}
\hline $\begin{array}{l}\text { Tratamento } \mathrm{P}_{2} \mathrm{O}_{5} \\
\left(\mathrm{~kg} \mathrm{ha}^{-1}\right)\end{array}$ & 0 & 150 & 300 & Média \\
\hline Calagem $^{/ 1}$ & \multicolumn{4}{|c|}{$\mathrm{mg} \mathrm{dm}^{-3} \longrightarrow$} \\
\hline $\mathrm{SC}$ & 7,01 & 16,18 & 35,55 & $19,58 \mathrm{a}$ \\
\hline $\mathrm{C} 0$ & 6,50 & 21,24 & 30,78 & $19,51 \mathrm{a}$ \\
\hline $\mathrm{C} 30$ & 8,96 & 18,19 & 29,58 & $18,91 \mathrm{a}$ \\
\hline Média & $7,49 \mathrm{c}$ & $18,54 \mathrm{~b}$ & $31,97 \mathrm{a}$ & \\
\hline $\mathrm{SC}$ & \multicolumn{3}{|c|}{ Amostragem 60 DAAP } & $6,42 \mathrm{a}$ \\
\hline $\mathrm{C} 0$ & 2,97 & 5,90 & 12,90 & $7,26 \mathrm{a}$ \\
\hline $\mathrm{C} 30$ & 2,77 & 4,52 & 14,90 & $7,40 \mathrm{a}$ \\
\hline \multirow[t]{2}{*}{ Média } & $2,66 \mathrm{c}$ & $4,86 \mathrm{~b}$ & $13,56 \mathrm{a}$ & \\
\hline & \multicolumn{3}{|c|}{ Amostragem 90 DAAP - } & 796 a \\
\hline C0 & 3,30 & 6,55 & 14,58 & $8,14 \mathrm{a}$ \\
\hline $\mathrm{C} 30$ & 3,21 & 5,65 & 13,87 & $7,58 \mathrm{a}$ \\
\hline \multirow[t]{2}{*}{ Média } & $2,97 \mathrm{c}$ & $5,89 \mathrm{~B}$ & $14,83 \mathrm{a}$ & \\
\hline & 2.29 & $\begin{array}{c}\text { Amostra } \\
6.74\end{array}$ & 20.38 & $9,80 \mathrm{a}$ \\
\hline $\mathrm{C} 0$ & 3,15 & 7,32 & 22,78 & $11,08 \mathrm{a}$ \\
\hline $\mathrm{C} 30$ & 3,70 & 7,17 & 17,10 & $9,32 \mathrm{a}$ \\
\hline Média & $3,05 \mathrm{c}$ & $7,08 \mathrm{~b}$ & $20,08 \mathrm{a}$ & \\
\hline
\end{tabular}

${ }^{1}$ Calagem, onde, $\mathrm{SC}=$ Sem calagem. $\mathrm{C} 0=$ Calagem realizada junto a aplicação do fósforo. $\mathrm{C} 30$ = Calagem realizada 30 dias antes da aplicação do fósforo. DAAP= Dias após aplicação do fósforo. * Médias seguida de letras minúsculas na coluna e maiúsculas na linha, não diferem entre si pelo teste de Tukey $(\mathrm{P}<0,05)$.

O RQo apresenta propriedades físicoquímicas que ocasionam em baixa capacidade de retenção de P. No entanto, em solos com pH mais elevado, com muito cálcio trocável, natural ou como consequência de uma supercalagem, pode ocorrer a precipitação de fosfatos adicionados ao solo (SAMPLE et al., 1980).

Nas extrações de fósforo pelo método Mehlich I foi observado que os valores de $\mathrm{P}$ disponível apresentaram mais elevados no RQo (Tabela 07) do que os valores obtidos para o LVdf (Tabela 03). Estes resultados ocorreram devido o RQo apresentar menores teores de argila total e matéria orgânica, o que condiciona em menor poder tampão e/ou tamponamente á esta classe de solo. De acordo com Novais et al., (2007) nos solos argilosos, o extrator é bem mais desgastado do que em solos mais arenosos.

O método da Resina de Troca Aniônica no RQo foi capaz de extrair maiores teores de $\mathrm{P}$ disponível nos tratamentos que receberam aplicação de $\mathrm{CaCO}_{3}$ (Tabela 8). Nas amostragens realizadas aos 60 DAAP e 120 DAAP foi constatada diferença significativa na interação entre as doses de $\mathrm{P}$ e as condições de calagem, já nas amostragens aos 30 DAAP e 90 DAAP não foi observada diferença significativa na interação, porém, os valores das médias das amostragens realizadas aos 90 DAAP foram superiores com uso do $\mathrm{CaCO}_{3}(\mathrm{C} 0$ e $\mathrm{C} 30)$.
Os maiores níveis de $\mathrm{P}$ com uso da Resina de Troca Aniônica no Neossolo Quartzarênico Órtico, mediante a prática da calagem, evidência de certa forma, a eficiência do processo de extração deste método em solos com textura Arenosa. De acordo com Souza et al., (2006) o fato do RQo apresentar proporções reduzidas da fração argila, propõe a esta classe de solos, características que se apresentam com menor capacidade de adsorção de $\mathrm{P}$, confirmando assim, os altos níveis deste elemento na solução do solo.

Os maiores teores de $\mathrm{P}$ resultaram do aumento do $\mathrm{pH}$ do solo com a prática da calagem. Visto que ocorre precipitação do $\mathrm{Al}$ e $\mathrm{Fe}$ da solução e redução da adsorção de íons fosfatos (CAMARGO et al., 2010). Todavia, de acordo com Haynes (1984) a retenção de $\mathrm{P}$ pelo solo pode permanecer com valores estequiométricos semelhantes, quando se substitui a precipitação do $\mathrm{P}$ pelo Alumínio trocável do solo ácido pela adsorção ao hidróxido de Al recém-formado pela calagem.

$\mathrm{Na}$ amostragem aos 120 DAAP, foi observado que o $\mathrm{CaCO}_{3}$ associado ao $\mathrm{P}$ (C0) apresentou maiores níveis de $\mathrm{P}$, condizendo com os maiores valores de $\mathrm{pH}$ também encontrados, mostrando que no decorrer do tempo e nestas condições de calagem (C0), o fósforo pode se tornar mais disponível na solução do solo. 
O método da resina, embora seja indicado para solos com amplas variações de textura, não exerce influências importantes nas propriedades químicas do solo, como a capacidade de troca de ânions (RAIJ, 1978) e a capacidade de extração de P disponível (SANTOS; KLIEMANN, 2005).

Entretanto, a utilização da Resina de Troca Aniônica na determinação do P disponível de um solo, corrige ou minimiza os problemas de subestimar ou superestimar os teores reais que o solo apresenta (RAIJ et al., 1986).

O P-rem não apresentou interação significativa entre as doses de $\mathrm{P}$ e as condições de calagem, entretanto, os valores das médias foram inferiores onde houve a aplicação de $\mathrm{CaCO}_{3}(\mathrm{CO}$ e C30), nas amostragens aos 30 DAAP e 60 DAAP
(Tabela 9). Isto, verificado nas primeiras amostragens pode está associado ao tempo curto das reações da solução do solo, indicando que, no decorrer das amostragens o $\mathrm{P}$ foi reagindo com o cálcio do $\mathrm{CaCO}_{3}$, diminuindo gradativamente os teores de P-rem.

Os elevados valores de P-rem obtidos no RQo ocorreram devido esta classe de solo apresentar menor capacidade de adsorção de $\mathrm{P}$, no entanto, se observarmos o LVdf, seus valores são bastante inferiores (Tabela 5), provavelmente, devido a maior capacidade de retenção de $\mathrm{P}$ que estes solos apresentam. A variação na adsorção de $\mathrm{P}$ entre os solos normalmente está associada à mineralogia, à textura e aos teores de matéria orgânica do solo (BAHIA FILHO et al., 1983).

Tabela 8. Teores de Fósforo (Resina) em diferentes condições de calagem e doses de $\mathrm{P}_{2} \mathrm{O}_{5}$, em Neossolo Quartzarênico Órtico no munícipio de Jataí-GO, 2010.

\begin{tabular}{|c|c|c|c|c|}
\hline $\begin{array}{l}\text { Tratamento } \mathrm{P}_{2} \mathrm{O}_{5} \\
\left(\mathrm{~kg} \mathrm{ha}^{-1}\right)\end{array}$ & 0 & 150 & 300 & Média \\
\hline Calagem $^{/ 1}$ & \multicolumn{4}{|c|}{$\mathrm{mg} \mathrm{dm}^{-3} \overline{\mathrm{DAAP}}$} \\
\hline $\mathrm{SC}$ & 22,52 & 50,37 & 72,14 & $48,34 \mathrm{a}$ \\
\hline $\mathrm{C} 0$ & 22,14 & 58,26 & 100,72 & $60,37 \mathrm{a}$ \\
\hline C30 & 36,88 & 43,21 & 121,06 & $67,05 \mathrm{a}$ \\
\hline \multirow[t]{2}{*}{ Média } & $27,18 \mathrm{C}$ & $50,61 \mathrm{~B}$ & 97,97 A & \\
\hline & \multicolumn{3}{|c|}{---- Amostragem 60 DAAP } & \\
\hline $\mathrm{SC}$ & $8,25 \mathrm{~b}$ B & $14,67 \mathrm{~b} \mathrm{~B}$ & $36,32 \mathrm{~b} \mathrm{~A}$ & $19,75 \mathrm{~b}$ \\
\hline $\mathrm{C} 0$ & 16,42 a C & 28,35 a B & 46,20 a A & $30,32 \mathrm{a}$ \\
\hline $\mathrm{C} 30$ & $13,15 \mathrm{ab} C$ & 20,75 b B & 51,75 a $\mathrm{A}$ & $28,55 \mathrm{a}$ \\
\hline \multirow{2}{*}{ Média } & $12,61 \mathrm{C}$ & $21,26 \mathrm{~B}$ & $44,76 \mathrm{~A}$ & \\
\hline & \multicolumn{3}{|c|}{ Amostragem 90 DAAP } & ------ \\
\hline $\mathrm{SC}$ & 9,46 & 18,86 & 42,35 & $23,56 \mathrm{~b}$ \\
\hline $\mathrm{C} 0$ & 15,50 & 25,54 & 53,60 & $31,55 \mathrm{a}$ \\
\hline $\mathrm{C} 30$ & 18,56 & 27,65 & 53,65 & $33,29 \mathrm{a}$ \\
\hline \multirow[t]{2}{*}{ Média } & $14,51 \mathrm{C}$ & $24,02 \mathrm{~B}$ & $49,87 \mathrm{~A}$ & \\
\hline & & ------ Amo & DAAP ------ & \\
\hline $\mathrm{SC}$ & $5,28 \mathrm{~b} \mathrm{C}$ & 15,76 c B & $38,69 \mathrm{c} \mathrm{A}$ & $19,91 \mathrm{c}$ \\
\hline $\mathrm{C} 0$ & 20,59 a C & 36,40 a B & 83,34 a A & $46,78 \mathrm{a}$ \\
\hline $\mathrm{C} 30$ & 13,30 a C & 26,58 b B & $60,67 \mathrm{~b} \mathrm{~A}$ & $33,52 \mathrm{~b}$ \\
\hline Média & $13,06 \mathrm{C}$ & $26,24 \mathrm{~B}$ & $60,90 \mathrm{~A}$ & \\
\hline
\end{tabular}

${ }^{1}$ Calagem, onde, $\mathrm{SC}=$ Sem calagem. $\mathrm{C} 0=$ Calagem realizada junto a aplicação do fósforo. $\mathrm{C} 30$ = Calagem realizada 30 dias antes da aplicação do fósforo. DAAP= Dias após aplicação do fósforo. * Médias seguida de letras minúsculas na coluna e maiúsculas na linha, não diferem entre si pelo teste de Tukey $(\mathrm{P}<0,05)$. 
Tabela 9. Teores de Fósforo Remanescente em diferentes condições de calagem e doses de $\mathrm{P}_{2} \mathrm{O}_{5}$, em Neossolo Quartzarênico Órtico no munícipio de Jataí-GO, 2010.

\begin{tabular}{|c|c|c|c|c|}
\hline $\begin{array}{l}\text { Tratamento } \mathrm{P}_{2} \mathrm{O}_{5} \\
\left(\mathrm{~kg} \mathrm{ha}^{-1}\right)\end{array}$ & 0 & 150 & 300 & Média \\
\hline \multicolumn{5}{|l|}{ Calagem $^{/ 1}$} \\
\hline $\mathrm{SC}$ & 35,92 & $\begin{array}{l}\operatorname{mostrag} \\
41,29\end{array}$ & 44,88 & $40,70 \mathrm{a}$ \\
\hline $\mathrm{CO}$ & 33,10 & 33,86 & 35,55 & $34,17 \mathrm{~b}$ \\
\hline C30 & 32,73 & 35,86 & 36,05 & $34,88 \mathrm{~b}$ \\
\hline Média & 33,92 B & $37,00 \mathrm{~A}$ & $38,83 \mathrm{~A}$ & \\
\hline \multicolumn{5}{|c|}{ - } \\
\hline $\mathrm{SC}$ & 43,90 & 46,07 & 50,72 & $46,90 \mathrm{a}$ \\
\hline $\mathrm{C} 0$ & 39,10 & 43,02 & 42,32 & $41,48 \mathrm{~b}$ \\
\hline $\mathrm{C} 30$ & 40,50 & 41,20 & 47,50 & $43,07 \mathrm{~b}$ \\
\hline Média & $41,17 \mathrm{~B}$ & $43,43 \mathrm{~B}$ & $46,85 \mathrm{~A}$ & \\
\hline $\mathrm{SC}$ & \multicolumn{4}{|c|}{ Amostragem 90 DAAP } \\
\hline $\mathrm{CO}$ & 41,34 & 42,17 & 43,06 & $42,19 \mathrm{a}$ \\
\hline $\mathrm{C} 30$ & 48,78 & 45,26 & 39,95 & $44,66 \mathrm{a}$ \\
\hline Média & $44,46 \mathrm{~A}$ & $44,27 \mathrm{~A}$ & $43,31 \mathrm{~A}$ & \\
\hline & 3780 & mostrage & 3813 & $3853 a$ \\
\hline $\mathrm{C} 0$ & $\begin{array}{l}37,89 \\
45,45\end{array}$ & $\begin{array}{l}39,55 \\
36,96\end{array}$ & $\begin{array}{l}38,13 \\
42,83\end{array}$ & $\begin{array}{l}38,53 \mathrm{a} \\
41,75 \mathrm{a}\end{array}$ \\
\hline $\mathrm{C} 30$ & 40,99 & 41,35 & 47,62 & $43,32 \mathrm{a}$ \\
\hline Média & $41,44 \mathrm{~A}$ & $39,29 \mathrm{~A}$ & $42,86 \mathrm{~A}$ & \\
\hline
\end{tabular}

${ }^{1}$ Calagem, onde, $\mathrm{SC}=$ Sem calagem. $\mathrm{C} 0=$ Calagem realizada junto a aplicação do fósforo. $\mathrm{C} 30=$ Calagem realizada 30 dias antes da aplicação do fósforo. DAAP= Dias após aplicação do fósforo. * Médias seguida de letras minúsculas na coluna e maiúsculas na linha, não diferem entre si pelo teste de Tukey $(\mathrm{P}<0,05)$.

\section{CONCLUSÕES}

Em ambos os solos, o extrator Mehlich I não foi eficiente em quantificar o teor de fósforo disponível com a prática da calagem.
O método Resina de Troca Aniônica mostrou-se eficiente na avaliação do teor de $\mathrm{P}$ disponível, mesmo com altas doses de Calcário aplicadas no solo.

\footnotetext{
ABSTRACT: Used a Oxisol and a Quartzipsament who received different doses of phosphorus (P) and limestone, with the objective of evaluating the content of available phosphorus in Mehlich I and Anion Exchange Resin in laboratory conditions. The experimental design was a randomized block in factorial consisting of two soils, three doses of phosphorus in the form of triple superphosphate $\left(0,150\right.$ and $\left.300 \mathrm{~kg} \mathrm{ha}^{-1}\right)$ and three conditions of liming (1. Limestone to 30 days before application of phosphorus. 2. Limestone along with application of phosphorus and, 3 . Without limestone) calculated to increase the base saturation to $70 \%$, with four repetitions, totaling 72 plots. The experiment lasted 150 days, ending the cycle of chemical reactions, with samplings at 30, 60, 90 and 120 days after application of phosphorus. The $\mathrm{pH}$, Mehlich-P, Resin-P and remaining-P. In both soils, the $\mathrm{pH}$ values increased to the use of limestone. In Oxisol levels of $\mathrm{P}$ extracted by Mehlich I were lower in treatments that were under liming practice being contrary to those obtained by the method of exchange resin anion, which proved superior to the application of lime. The RQo showed results similar to RH, showing that the extraction with anion exchange resin more efficiently detects increases in the levels of available phosphorus before liming.
}

KEYWORDS: Adsorption. Limestone. Incubations. Extractors.

\section{REFERÊNCIAS}

ALMEIDA, J. A.; TORRENT, J.; BARRÓN, V. Cor de solo, formas do fósforo e adsorção de fosfato em Latossolos desenvolvidos de basalto do extremo sul do Brasil. Revista Brasileira de Ciência do Solo, v. 27, n. 6, p. 985-1002, 2003. http://dx.doi.org/10.1590/S0100-06832003000600003 
ALVAREZ, V. V. H.; NOVAIS, R. F.; DIAS, L. E.; OLIVEIRA, J. A. Determinação e uso do fósforo remanescente. Boletim informativo da Sociedade Brasileira de Ciência do Solo, Viçosa, v. 25, n. 1, p. 27 32,2000 .

ALVAREZ, V. V. H.; FONSECA, D. M. Definição de doses de fósforo para determinação da capacidade máxima de adsorção de fosfatos e para ensaios em casa de vegetação. Revista Brasileira de Ciência do Solo, Campinas, v. 14, p. 49-55, 1990.

BAHIA FILHO, A. F. C.; BRAGA, J. M.; RIBEIRO, A. C.; NOVAES, R. F. Sensibilidade de extratores químicos à capacidade tampão de fósforo. Revista Brasileira de Ciência do Solo, Campinas, v. 7, n. 2, p. 243$249,1983$.

BARROW, N. J. Reaction of anions and cations with variable-charge soils. Advances in Agronomy, 38:183230, 1985. http://dx.doi.org/10.1016/S0065-2113(08)60676-8

BECKETT, P. H. T.; WHITE, R. E. Studies on the phosphate potentials of soils. III. The pool of labile inorganic phosphate. Plant and Soil, The Hague, v. 21, p. 253-282, 1964.

http://dx.doi.org/10.1007/BF01377744

CAMARGO, M. S; BARBOSA, D. S; RESENDE, R. H; KORNDÖRFER, G. H; PEREIRA, H. S. Fósforo em solos de cerrado submetidos a calagem. Bioscience Journal, v. 26, n. 2, p. 187-194, 2010.

EMPRESA BRASILEIRA DE PESQUISA AGROPECUÁRIA - EMBRAPA. Sistema Brasileiro de classificação de Solos. 3. ed. Rio de Janeiro: EMBRAPA Solos, 2013. 350 p.

FERREIRA, D. F. SISVAR: um programa para análises e ensino de estatística. Revista Symposium, Campinas, v. 6, p. 36-41, 2008.

GATIBONI, L. C. et al. Superphosphate and rock phosphates as Phosphorus sources for grass-clover pasture on a limed acid soil in Southern Brazil. Communications in Soil Science and Plant Analysis, v. 34, n. 17/18, p. 2503-2514, 2003. http://dx.doi.org/10.1081/CSS-120024782

HAYNES, R. J. Lime and phosphate in the soil-plant system. Advances in Agronomy, v. 37, p. 249-315, 1984. http://dx.doi.org/10.1016/S0065-2113(08)60456-3

LARSEN, S. Soil phosphorus. Advances in Agronomy, New York, v. 19, p. 151-210, 1967. http://dx.doi.org/10.1016/S0065-2113(08)60735-X

MEHLICH, A. New extractant for soil test evaluation of phosphorus, potassium, magnesium, calcium, sodium, manganese and zinc. Communications in Soil Science and Plant Analysis, New York, v. 9, n. 6, p. 477-492, 1978. http://dx.doi.org/10.1080/00103627809366824

NOVAIS, R. F.; SMITH, T. J. Fósforo em solo e planta em condições tropicais. UFV: Viçosa, 1999, 399 p.

NOVAIS, R. F.; SMYTH, T. J.; NUNES, F. N.; Fósforo. In: NOVAIS, R. F.; ALVAREZ, V. H. V.; BARROS, N. F.; FONTES, R. L. F.; CANTARUTTI, B. B.; NEVES, J. C. L. Fertilidade do Solo. Viçosa: Sociedade Brasileira de Ciência do Solo, 2007. Cap. VIII. p. 472-552.

OLSEN, S. R.; KHASAWNEH, F. E. Use and limitations of physical-chemical criteria for assessing the status of phosphorus in soils. In: KHASAWNEH, F. E.; SAMPLES, E. C.; KAMPRATH, E. J. The role of phosphorus in agriculture. Madison, American Society of Agronomy, p. 361-410. 1980.

RAIJ, B. V. Seleção de métodos de laboratório para avaliar a disponibilidade de fósforo em solos. Revista Brasileira de Ciência do Solo, Campinas, v. 2, n. 1, p. 1-9, 1978. 
RAIJ, B. V.; FEITOSA, C. T.; SILVA, N. M. Comparação de quatro extratores de solos. Bragantia, Campinas, v. 43, n. 1, p. 17-29, 1984. http://dx.doi.org/10.1590/S0006-87051984000100003

RAIJ, B. V; QUAGGIO, J. A.; SILVA, N. M. Extraction of phosphorus, potassium, calcium, and magnesium from soils by an ion-exchange resin procedure. Communications in Soil Science and Plant Analysis, New York, v. 17, n. 5, p. 547-566, 1986. http://dx.doi.org/10.1080/00103628609367733

RAIJ, B. V; QUAGGIO, J. A. Extractable phosphorus availability indexes as affected by liming. Communications in Soil Science and Plant Analysis, New York, v. 21, n. 13/16, p. 1267-1276, 1990. http://dx.doi.org/10.1080/00103629009368304

ROSSI, C.; FAGUNDES, J. L. Determinação do teor de fósforo em solos por diferentes extratores. Revista de Agricultura, Piracicaba, v. 73, n. 2, p. 215-227, 1998.

SAMPLE, E. C.; SOPER, R. J.; RACZ, G. J. Reactions of phosphate in soils. In.: KHASAWNEH, F. E.; SAMPLE, E. C.; KAMPRATH, E. J. (Ed.). The role of phosphorus in agriculture. Madison: American Society of Agronomy, chap. 11, p. 263-310, 1980.

SANTOS, E. A.; KLIEMANN, H. J. Disponibilidade de fósforo de fosfatos naturais em solos de cerrado e sua avaliação por extratores químicos. Pesquisa Agropecuária Tropical, v. 35, n. 3, p. 139-146, 2005.

SILVA, F. C.; RAIJ, B. V. Disponibilidade de fósforo em solos avaliadas por diferentes extratores. Pesquisa Agropecuária Brasileira, Brasília, v. 34, n. 2, p. 267-288, 1999. http://dx.doi.org/10.1590/S0100204X1999000200016

SOUZA, R. F.; FAQUIN, V.; TORRES, P. R. F.; BALIZA, D. P. Calagem e adubação orgânica: influência na adsorção de fósforo em solos. Revista Brasileira de Ciência do Solo, v. 30, p. 975-983, 2006. http://dx.doi.org/10.1590/S0100-06832006000600007

SOUSA, D. M. G.; LOBATO, E.; REIN, T. A. Adubação com fósforo. In: SOUSA, D. M. G.; LOBATO, E., eds. Cerrado: Correção do solo e adubação. 2.ed. Planaltina, Embrapa Cerrados, p.147-168. 2004.

SANTOS, D. B. M. Distribuição do fósforo no perfil do solo sob sistema plantio direto. 2009. $81 \mathrm{f}$. Dissertação (Mestrado) - Faculdade de Agronomia e Medicina Veterinária, Universidade de Brasília, Brasília, 2009. 